\title{
Влияние низкодозного протонного облучения на характеристики инжекционных диодов на основе $4 \mathrm{H}$-SiC
}

\author{
(С) П.А. Иванов, А.С. Потапов, М.Ф. Кудояров, Т.П. Самсонова \\ Физико-технический институт им. А.Ф. Иоффре Российской академии наук, \\ 194021 Санкт-Петербург, Россия \\ E-mail: Pavel.Ivanov@mail.ioffe.ru
}

(Получена 12 марта 2018 г. Принята к печати 22 марта 2018 г.)

\begin{abstract}
Исследовано влияние низкодозного протонного облучения (доза облучения $10^{10}-1.8 \cdot 10^{11} \mathrm{~cm}^{-2}$ ) на вольтфарадные характеристики, прямые вольт-амперные характеристики и характеристики обратного восстановления высоковольтных диодов с $p-n_{o}$-переходом на основе $4 H$-SiC. Облучение проводилось протонами с энергией $1.8 \mathrm{MэB} \mathrm{через} \mathrm{никелевую} \mathrm{пленку} \mathrm{толщиной} 10$ мкм (энергия протонов и толщина Ni пленки выбирались так, чтобы пробег протонов в карбиде кремния приблизительно равнялся глубине залегания $p-n_{o}$-перехода). Показано, что протонное облучение в указанных дозах: 1) не вызывает сколько-нибудь заметного уменьшения концентрации основных носителей в диодах; 2) кардинально уменьшает время жизни неравновесных носителей при низком уровне инжекции (в десятки раз при максимальной дозе облучения); 3) уменьшает заряд обратного восстановления диодов при высоком уровне инжекции (до трех раз при максимальной дозе облучения).
\end{abstract}

DOI: 10.21883 /FTP.2018.10.46459.8863

\section{1. Введение}

Облучение высокоэнергетическими частицами (электронами, $\alpha$--частицами, протонами) широко применяется для коррекции динамических характеристик кремниевых инжекционных $p-n$-диодов (см., например, [1]). (Облучение высокоэнергетичными частицами позволяет изменять профиль времени жизни инжектированных неосновных носителей в диодах и тем самым регулировать характеристики обратного восстановления). Что касается карбида кремния, то до настоящего времени экспериментальных работ в этом направлении не проводилось. В работах [2,3] было численно промоделировано (в среде SILVACO TCAD) влияние облучения протонами c энергией 670 кэВ на статические и динамические характеристики высоковольтных $p^{+}-n^{-}-n^{+}$-диодов на основе 4H-SiC. В цитированных теоретических работах доминирующими рекомбинационными ловушками были приняты известные центры $Z 1 / Z 2$, концентрация которых растет при облучении протонами [4]. (Стоит отметить, что концентрация ловушек $Z 1 / Z 2$ растет при облучении не только протонами, но и другими частицами, например электронами [5]). Моделирование показало, что характеристики обратного восстановления должны быть чувствительны к малым дозам облучения, начиная от $10^{10} \mathrm{~cm}^{-2}$. В настоящей работе нами впервые проведены экспериментальные исследования влияния такого низкодозного $\left(10^{10}-1.8 \cdot 10^{11} \mathrm{~cm}^{-2}\right)$ протонного облучения на характеристики $4 H$-SiC-диодов с высоковольтным (свыше 2 кВ) $p-n_{o}$-переходом.

\section{2. Образцы}

Для изготовления диодов была использована коммерческая $4 H-\mathrm{SiC}$ пластина с многослойной эпитаксиальной структурой: $p^{++}-p^{+}-p-n_{o}-n^{+}-n_{s}$ (подложка). Концентрации примесей в $p^{++}-, p^{+}, p-, n_{o^{-}}$и $n^{+}$-слоях составляют $2 \cdot 10^{19}, 5 \cdot 10^{18}, 4 \cdot 10^{16}, 5 \cdot 10^{15}$ и $1 \cdot 10^{18} \mathrm{~cm}^{-3}$ соответственно. Толщины $p^{++}-, p^{+},, p-, n_{0^{-}}$и $n^{+}$-слоев составляют $0.5,0.5,6,20$ и 5 мкм соответственно.

Диоды изготавливались в следующей последовательности. Вначале на обратной стороне пластины был сформирован омический контакт: слой никеля толщиной 0.1 мкм осаждался магнетронным распылением с последующим термическим отжигом при температуpe $950^{\circ} \mathrm{C}$ в течение получаса. Затем на поверхности эпитаксиальной структуры были сформированы омические контакты площадью $S=2.5 \cdot 10^{-2} \mathrm{~cm}^{2}$ : контактный слой, состоящий из алюминия (0.07 мкм) и титана (0.03 мкм), осаждался электронно-лучевым напылением

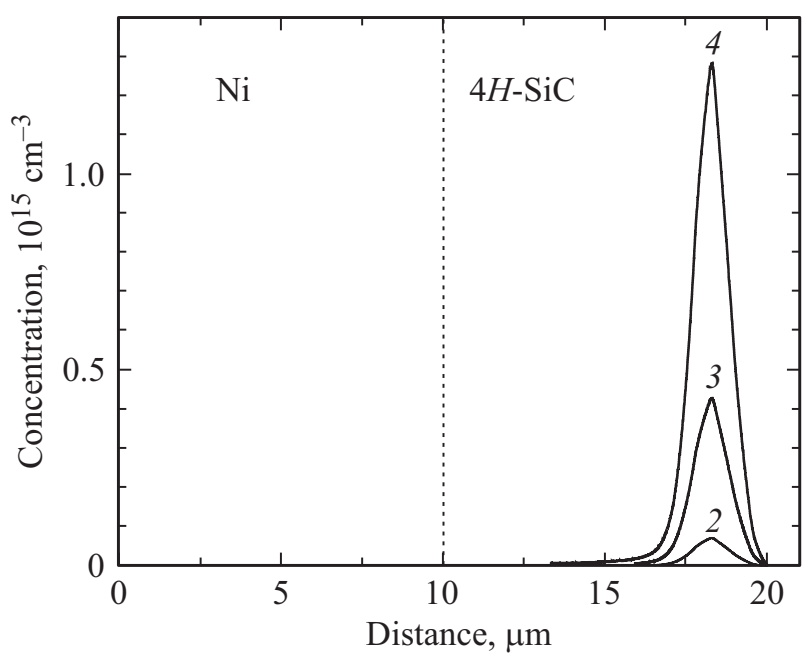

Рис. 1. Концентрационные профили распределения $1.8 \mathrm{MэВ}$ протонов, внедренных в $4 H$-SiC-диоды 2,3 и 4 (расчет по программе SRIM). 
с последующим термическим отжигом при температуре $950^{\circ} \mathrm{C}$ в течение 5 мин. Поверх контактной металлизации гальваническим осаждением был нанесен маскирующий слой никеля толщиной около 10 мкм. Далее на обратную сторону пластины был нанесен слой никеля толщиной 1 мкм магнетронным распылением. Диоды были выполнены в виде мезаструктур, сформированных сухим селективным травлением $4 H$-SiC на глубину около 10 мкм. Пластина с дискретными диодами разрезалась на отдельные чипы.

Контрольные диоды (диоды 1 ) облучению не подвергались. Протонное облучение проводилось на УНУ „Циклотрон ФТИ“. Энергия протонов (1.8 МэВ) и толщина маскирующего слоя Ni 10 мкм) выбирались так, чтобы пробег протонов в карбиде кремния приблизительно равнялся глубине залегания $p-n_{o}$-перехода (рис. 1). Доза облучения варьировалась: $1 \cdot 10^{10} \mathrm{~cm}^{-2}$ (диоды 2), $6 \cdot 10^{10} \mathrm{~cm}^{-2}$ (диоды 3) и $1.8 \cdot 10^{11} \mathrm{~cm}^{-2}$ (диоды 4). После облучения диоды температурной обработке не подвергались.

\section{3. Экспериментальные результаты и обсуждение}

Измерения вольт-фарадных характеристик (ВФХ) изготовленных диодов проводились с помощью цифрового измерителя иммиттанса E7-20. На рис. 2 показаны типичные ВФХ диодов 1-4. Как видно, измеренные ВФХ практически совпадают, что свидетельствует о том, что низкодозное протонное облучение не оказывает сколько-нибудь заметного компенсирующего влияния на концентрацию основных носителей в базовых областях диодов.

На рис. 3 точками показаны типичные прямые вольтамперные характеристики (BAX) диодов $1-4$, измеренные при низких плотностях тока и построенные в полулогарифмическом масштабе: $\log (j)=f(V)$. Показанные ВАХ имеют классический вид [6]. Они состоят

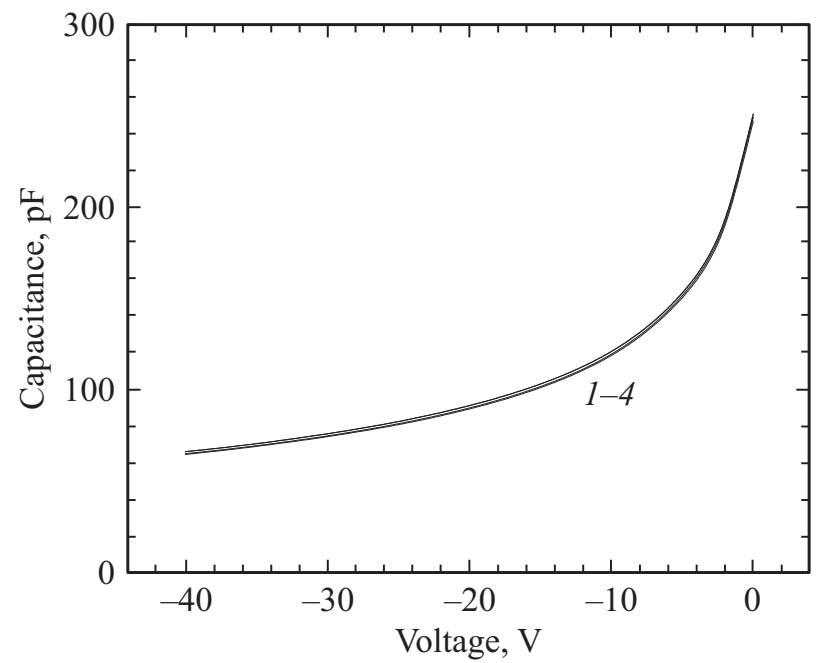

Рис. 2. ВФХ 4H-SiC-диодов $1-4$, измеренные на частоте 1 кГц.

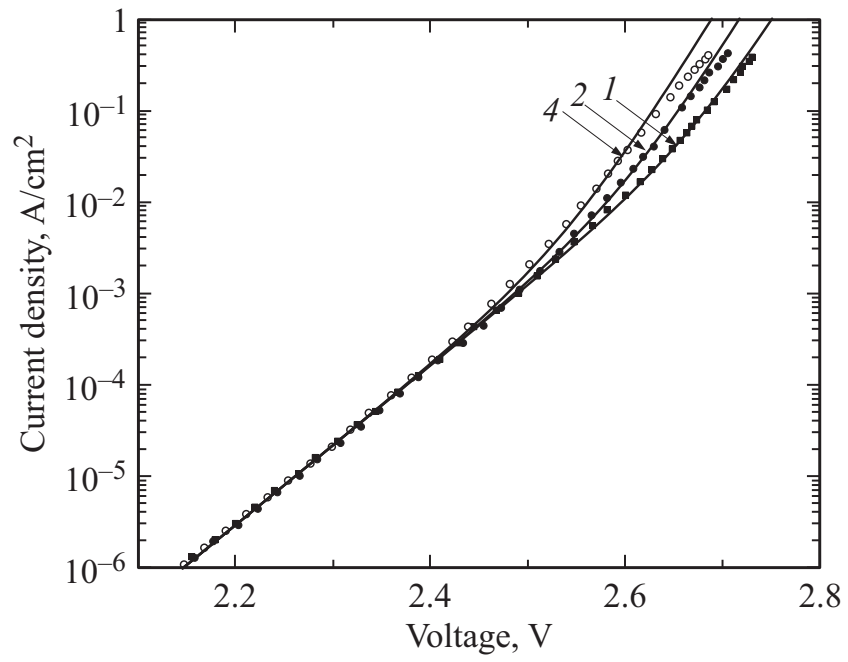

Рис. 3. $\mathrm{BAX} 4 H$-SiC-диодов 1,2 и 4, измеренные при низких плотностях тока и построенные в полулогарифмическом масштабе. Точки - экспериментальные данные, сплошные линиии - аппроксимации экспериментальных данных суммой рекомбинационного и диффузионного токов.

из трех участков: на первом участке доминирует рекомбинационный ток в области пространственного заряда (ОПЗ) $p-n_{o}$-перехода; на втором участке к рекомбинационному току в ОПЗ добавляется диффузионный ток в нейтральной части базовых областей; на третьем участке происходит переход к режиму ограничения тока последовательным сопротивлением диода. На первом участке зависимости $j(V)$ для всех диодов $1-4$ практически совпадают (облучение не создает дополнительных рекомбинационных центров в середине запрещенной зоны $4 H-\mathrm{SiC})$. На втором участке наблюдается явное увеличение диффузионной компоненты тока, обусловленное уменьшением времени жизни инжектированных носителей в базовых областях. На рис. 3 сплошными линиями показаны аппроксимации экспериментальных данных (на первом и втором участках ВАХ) суммой рекомбинационного $\left(j_{\text {rec }}\right)$ и диффузионного $\left(j_{\text {diff }}\right)$ токов:

$$
j=j_{\text {rec }}+j_{\text {diff }}=j_{\text {or }} \exp (q V / 2 k T)+j_{\text {od }} \exp (q V / k T),
$$

где $q-$ элементарный заряд, $k T-$ тепловая энергия. Предэкспоненциальный множитель $j_{\text {or }}$ (рекомбинационная составляющая плотности тока насыщения) определялся экспериментально. Множитель $j_{\text {od }}$ (диффузионная составляющая плотности тока насыщения) был подгоночным параметром для наилучшей аппроксима-

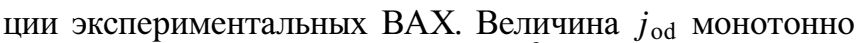
увеличивается от $1.3 \cdot 10^{-48} \mathrm{~A} / \mathrm{cm}^{2}$ для необлученных диодов до $1.8 \cdot 10^{-47} \mathrm{~A} / \mathrm{cm}^{2}$ для диодов, облученных максимальной дозой. Как мы полагаем, в исследуемых диодах основной вклад в диффузионный ток должна вносить электронная компонента (диффузия электронов в $p$-области). В этом случае параметр $j_{\text {od }}$ рассчитывается 
следующим образом [7]:

$$
j_{\mathrm{od}}=\frac{q n_{i}^{2}}{p_{p}} \sqrt{\frac{D_{n}}{\tau_{n}}}
$$

где $n_{i}$ - концентрация собственных носителей в $4 H-\mathrm{SiC}$, $p_{p}$ - концентрация дырок в $p$-области, $\overline{D_{n}}-$ коэффициент диффузии электронов и $\tau_{n}-$ время жизни электронов в $p$-области. Для необлученных диодов расчет по формуле (2) дает время жизни электронов около 3 нс. Если считать, что облучение не оказывает влияния на концентрацию основных носителей (дырок), то тогда следует полагать, что облучение кардинально уменьшает время жизни электронов (в несколько десятков раз при максимальной дозе облучения). Вследствие усиления диффузионной компоненты тока в $p$-области уменьшается коэффициент инжекции $p-n_{o}$-перехода, так что следует ожидать улучшения динамических характеристик облученных диодов.

Действительно, измерения характеристик обратного восстановления $(\mathrm{OB})$ изготовленных диодов продемонстрировали существенное улучшение быстродействия диодов после облучений (на рис. 4 показаны типичные характеристики ОВ диодов $1-4$ при их переключении с прямого тока $3 \mathrm{~A}$ на обратное напряжение $500 \mathrm{~B}$ ). Помимо сравнения величин зарядов ОВ, определяемых как интеграл от обратного тока по времени, было интересно сравнить эти заряды с зарядом $Q_{n}$ основных носителей, выносимых из $n_{o}$-базы емкостным током:

$$
Q_{n}=q N_{d} W S=21 \text { нКл, }
$$

где $N_{d}=5 \cdot 10^{15} \mathrm{~cm}^{-3}-$ концентрация доноров в $n_{o}$-базе, $W=10.5$ мкм - ширина ОПЗ в $n_{o}$-базе при обратном напряжении $500 \mathrm{~B}$. Отметим, что заряд ОВ необлученных диодов 1 (129нКл) оказался больше $Q_{n}$ в 6 раз. Заряд ОВ диодов 2 (74 нКл) уменьшается по сравнению с необлучеными диодами в 1.7 раза.

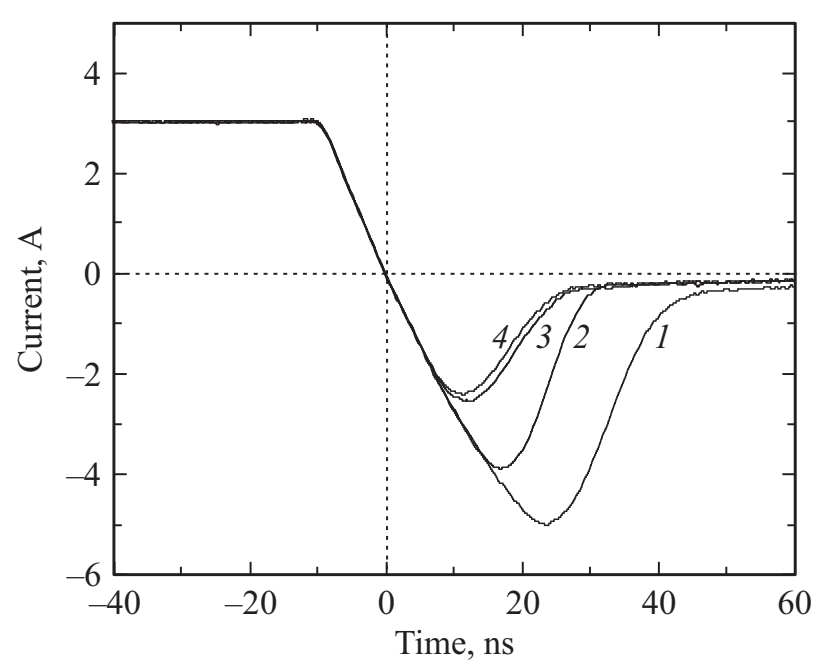

Рис. 4. Характеристики обратного восстановления $4 H-\mathrm{SiC}$ диодов 1-4. Прямой ток $3 \mathrm{~A}$, обратное напряжение $500 \mathrm{~B}$.

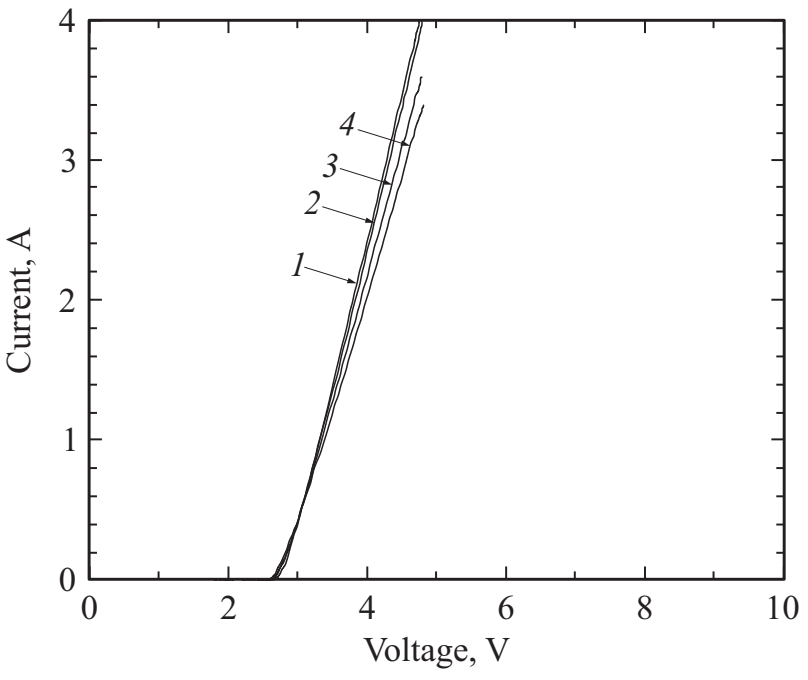

Рис. 5. BAX $4 H$-SiC-диодов $1-4$, измеренные с помощью характериографа Л2-100.

Заряды ОВ диодов 3 и 4 (48 и 43 нКл соответственно) незначительно отличаются между собой и в 3 раза меньше по сравнению с необлученными диодами. Отметим, что после облучения диоды сохраняют „мягкий“ характер восстановления, что косвенно подтверждает высказанное ранее предположение о том, что облучение приводит, главным образом, к снижению коэффициента инжекции $p-n_{o}$-перехода.

На рис. 5 показаны типичные ВАХ диодов 1-4, измеренные при токах до $3 \mathrm{~A}$ (измерения проводились с помощью цифрового характериографа Л2-100 в режиме однократного запуска развертки по напряжению). Напряжение открывания всех диодов одинаково и составляет около $2.8 \mathrm{~B}$ (эта величина приблизительно равна контактной разности потенциалов $4 H-\mathrm{SiC} p-n$-перехода). Как видно, существенного влияния облучения на дифференциальное сопротивление диодов в прямом направлении нет (дифференциальное сопротивление исследованных диодов в открытом состоянии $\left(1.2 \cdot 10^{-3} \mathrm{OM} \cdot \mathrm{cm}^{2}\right)$ определяется главным образом сопротивлением омических контактов). Таким образом, низкодозное облучение протонами вполне может стать эффективным инструментом для улучшения динамических характеристик $4 H$-SiC-диодов без значительного ухудшения их статических параметров.

\section{4. Заключение}

В заключение можно отметить следующее. В настоящее время в силовой и импульсной электронике уже широко используются высоковольтные (до 1700 В) диоды Шоттки на основе $4 H$-SiC. Быстро восстанавливающиеся инжекционные $4 H$-SiC-диоды тоже могут найти свою нишу в силовой электронике (благодаря большей рабочей температуре перехода и более высокой надежности), а также в субнаносекундной импульсной технике в качестве дрейфовых диодов с резким восстановлением [8]. 
Проведенное в настоящей работе экспериментальное исследование показало возможность эффективной коррекции динамических характеристик инжекционных $4 H$-SiCдиодов с помощью низкодозного протонного облучения. Важной отличительной особенностью $4 H-\mathrm{SiC}$ диодов является то, что базовые области высоковольтных (более 1 кВ) диодов имеют сравнительно высокую концентрацию легирующих примесей, превышающую, как правило, $5 \cdot 10^{15} \mathrm{~cm}^{-3}$. В результате прямые вольтамперные характеристики будут, по-видимому, менее чувствительны к низкодозному облучению по сравнению, например, с кремниевыми диодами. В дальнейшем предстоит детально исследовать весь спектр проблем, связанных с радиационной коррекцией характеристик инжекционных 4H-SiC-диодов. К ним относятся: определение параметров радиационных дефектов при разных видах облучений, исследования пространственных распределений дефектов и их термической устойчивости, дальнейшие исследования влияния облучений не только на время обратного восстановления диодов, но и на характер обрыва обратного тока в них.

Исследование выполнено за счет гранта Российского научного фонда (проект № 14-29-00094).

\section{Список литературы}

[1] P. Hazdra, J. Vobecký, H. Dorschner, K. Brand. Microelectron. J., 35, 249 (2004).

[2] R.K. Sharma, P. Hazdra, S. Popelka. Int. J. Microelectron. Comput. Sci., 6, 59 (2015).

[3] P. Hazdra, S. Popelka. Mater. Sci. Forum, 897, 463 (2017).

[4] R.K. Sharma, S. Popelka, P. Hazdra. IEEE Trans. Nucl. Sci., 62, 534 (2015)

[5] K. Danno, D. Nakamura, T. Kimoto. Appl. Phys. Lett., 90, 202109 (2007).

[6] P.A. Ivanov, M.E. Levinshtein, J.W. Palmour, S.L. Rumyantsev, R. Singh. Semicond. Sci. Technol., 15, 908 (2000).

[7] С. Зи. Физика полупроводниковых приборов (М., 1984) т. 1.

[8] П.А. Иванов, О.И. Коньков, Т.П. Самсонова, А.С. Потапов. Письма ЖТФ, 44, 3 (2018).

Редактор А.Н. Смирнов

\section{The effect of low dose proton irradiation on electrical characteristics of $4 \mathrm{H}$-SiC junction diodes}

\author{
P.A. Ivanov, A.S. Potapov, V.F. Kudoyarov, \\ T.P. Samsonova \\ loffe Institute, \\ 194021 St. Petersburg, Russia
}

Abstract Capacitance-voltage, current-voltage and reverse recovery characteristics of $4 \mathrm{H}$-SiC junction diodes irradiated with protons (irradiation dose $10^{10}-1.8 \cdot 10^{11} \mathrm{~cm}^{-2}$ ) have been investigated. Irradiations with $1.8 \mathrm{MeV}$ protons were done through $10 \mu \mathrm{m}$ thick Ni-film (the energy of protons and thickness of Ni-film were chosen to ensure the projected range of protons in silicon carbide approximately equal to the junction depth). After proton irradiations: 1) the concentrations of majority carriers are not changed; 2) the lifetime of minority carriers (at low injection level) is drastically reduced (several ten times at the highest irradiation dose); 3 ) the reverse recovery charge is reduced (3 times at the highest irradiation dose). 